\title{
Early Lung Cancer Detection Using Spiral Computed Tomography and Positron Emission Tomography
}

\author{
Gorka Bastarrika, María José García-Velloso, Maria Dolores Lozano, Usua Montes, Wenceslao Torre, \\ Natalia Spiteri, Arantza Campo, Luis Seijo, Ana Belén Alcaide, Jesús Pueyo, David Cano, Isabel Vivas, \\ Octavio Cosín, Pablo Domínguez, Patricia Serra, José A. Richter, Luis Montuenga, and Javier J. Zulueta
}

Departments of Radiology, Nuclear Medicine, Pathology, Pulmonary Medicine, and Thoracic Surgery, Clínica Universitaria; Department of Histology and Pathology, School of Medicine; and Division of Oncology, Center for Applied Medical Research (CIMA), Universidad de Navarra, Pamplona, Spain

\begin{abstract}
Rationale: Lung cancer screening using computed tomography (CT) is effective in detecting lung cancer in early stages. Concerns regarding false-positive rates and unnecessary invasive procedures have been raised. Objective: To study the efficiency of a lung cancer protocol using spiral CT and F-18-fluorodeoxyglucose positron emission tomography (FDG-PET). Methods: High-risk individuals underwent screening with annual spiral CTs. Follow-up CTs were done for noncalcified nodules of $5 \mathrm{~mm}$ or greater, and FDG-PET was done for nodules $10 \mathrm{~mm}$ or larger or smaller $(>7 \mathrm{~mm})$, growing nodules. Results: A total of 911 individuals completed a baseline CT study and 424 had at least one annual follow-up study. Of the former, $14 \%$ had noncalcified nodules of $5 \mathrm{~mm}$ or larger, and $3.6 \%$ had nodules of $10 \mathrm{~mm}$ or larger. Eleven non-small cell lung cancers (NSCLC) and one small cell lung cancer (SCLC) were diagnosed in the baseline study (prevalence rate, $1.32 \%$ ), and two NSCLCs in the annual study (incidence rate, $0.47 \%$ ). All NSCLCs $(92 \%$ of prevalence cancers) were diagnosed in stage I (12 stage IA, 1 stage IB). FDG-PET was helpful for the correct diagnosis in 19 of 25 indeterminate nodules. The sensitivity, specificity, positive predictive value, and negative predictive value of FDG-PET for the diagnosis of malignancy were $69,91,90$, and $71 \%$, respectively. However, the sensitivity and negative predictive value of the screening algorithm, which included a 3-month follow-up CT for nodules with a negative FDGPET, was $100 \%$. Conclusion: A protocol for early lung cancer detection using spiral CT and FDG-PET is useful and may minimize unnecessary invasive procedures for benign lesions.
\end{abstract}

Keywords: chronic obstructive pulmonary disease; lung neoplasm; pulmonary nodule; smoking

Lung cancer is the most frequent and lethal malignancy in the world (1-3). Advances in the treatment of locally advanced lung cancer have had no impact on overall 5-year survival rates from this disease $(1,2)$. In countries where gains against tobacco smoking have been made, mortality rates from lung cancer have started to decrease, but future projections are not optimistic because of the recent surge in tobacco consumption among young people (4). And even if smoking could be reduced significantly, the long lag time between peak tobacco consumption and the development of clinical lung cancer will assure a long life for this epidemic.

The main reason why more than $80 \%$ of patients with lung cancer die soon after diagnosis is that most patients are diag-

(Received in original form November 5, 2004; accepted in final form March 18, 2005) Correspondence and requests for reprints should be addressed to Javier J. Zulueta, M.D., Pulmonary Medicine, Clínica Universitaria, Avda. Pio XII, 36, 31008 Pamplona, Spain. E-mail: jzulueta@unav.es

This article has an online supplement, which is accessible from this issue's table of contents at www.atsjournals.org

Am J Respir Crit Care Med Vol 171. pp 1378-1383, 2005

Originally Published in Press as DOI: 10.1164/rccm.200411-14790C on March 24, 2005

Internet address: www.atsjournals.org nosed in late nonsurgical stages (4). Large screening trials have investigated the use of conventional radiology and sputum cytology for early detection of lung cancer but failed to show a reduction in disease-specific mortality, resulting in public policies against screening for this disease (5-8). However, recently, very promising reports using spiral computed tomography (CT) have brought lung cancer screening back to the forefront. The Early Lung Cancer Action Project (ELCAP) demonstrated that lowdose spiral CT detects lung cancer in early stages much more effectively than conventional chest radiograph (9). Following a carefully designed protocol based on follow-up CTs to detect growth of small nodules, $86 \%$ of lung cancers diagnosed in asymptomatic individuals were in stage I (9). Other studies using spiral CT have found similar results, although with variations in the percentage of individuals with positive CTs (i.e., with noncalcified nodules that need further workup) (10-14). Swensen and colleagues (10) reported noncalcified nodules in close to $70 \%$ of the individuals participating in a lung cancer screening trial with spiral CT. Diederich and coworkers (11) found noncalcified nodules in $43 \%$ of their population. Although the ELCAP investigators only performed one invasive procedure on one benign lesion (9), the high prevalence of noncalcified nodules may result in unnecessary invasive procedures for benign diseases. This will be of particular concern when these protocols become widely used in the community with less rigorous standards. Positron emission tomography with the glucose analog F-18-fluorodeoxyglucose (FDG-PET) is an accurate noninvasive imaging test for diagnosis of pulmonary nodules, although few data exist for nodules smaller than $1 \mathrm{~cm}$ in diameter (15). Pastorino and colleagues (12) showed that selective use of FDGPET in a spiral CT-based lung cancer screening trial may be useful in avoiding biopsies for benign lesions. The hypothesis formulated in the study presented herein is that adding FDGPET to a spiral CT-based early lung cancer detection protocol will avoid unnecessary procedures for benign lesions. Furthermore, false-negative lesions (i.e., cancers with no pathologic uptake on the FDG-PET) will be diagnosed with follow-up CTs to detect growth, and therefore such a protocol will not result in missed cancers because of negative PET scans.

After publication of their original results, the ELCAP group led two important initiatives: the NY-ELCAP screening trial, a New York-area multicenter trial projecting to screen 10,000 high-risk individuals with spiral CT, and the I-ELCAP, an international consortium that brings together investigators from over 30 centers from around the world (16). One of the main objectives of I-ELCAP is to pool data from screening trials underway in these centers. The current article presents results from an ongoing trial of lung cancer screening using spiral CT and FDG-PET conducted at our institution as a member of I-ELCAP since 2000. Some of the results of these studies have been previously reported in the form of an abstract (17). 


\section{METHODS}

\section{Population}

Current and former smokers of at least 40 years of age, with a minimum 10 pack-year smoking history, and with no symptoms of lung cancer were invited to participate. A background questionnaire and written, informed consent were obtained. The study protocol was approved by our center's ethics committee.

\section{CT}

CT examinations were done in a single breath-hold at end-inspiration. The initial 297 subjects were studied with a single-slice helical scanner (Somatom Plus 4; Siemens, Erlangen, Germany) at low-dose settings (140 kilovolt peak [kVp], 43 milliampere seconds [mAs]) and 1.5 pitch with a collimation of $8 \mathrm{~mm}$. Pulmonary nodules were characterized with a limited high-resolution CT (1-mm slice thickness). For the subsequent 614 patients, a four-row multislice helical CT scanner was used (Somatom Volume Zoom; Siemens, Forchheim, Germany) at a lowdose setting (120 kVp, 20 mAs, 1.25-mm slice thickness). Studies were analyzed independently by two radiologists.

\section{Pulmonary Nodules}

Calcifications, size, location, edges, and spiculations were documented for each nodule. Size was the average of length and width and classified nodules into three categories: less than $5 \mathrm{~mm}, 5$ to but not including $10 \mathrm{~mm}$, and $10 \mathrm{~mm}$ or greater.

\section{FDG-PET}

Except for nodules with benign characteristics, noncalcified nodules of $10 \mathrm{~mm}$ or greater, or smaller nodules $(>7 \mathrm{~mm})$ showing growth, were evaluated with FDG-PET. Imaging acquisition was performed with a full ring bismuth germanate (BGO) tomograph (Ecat Exact HR +; CTI/ Siemens, Erlangen, Germany). Visual analysis was performed by two nuclear medicine physicians, and was considered positive for malignancy when focal uptake was observed in the lung nodule. Metabolic activity was assessed by the maximum standardized uptake value (SUVmax).

\section{Diagnostic Algorithm}

The result of the baseline screening test was considered positive if there were one to six noncalcified nodules, or more than six nodules with the largest one being $5 \mathrm{~mm}$ or greater. When the initial low-dose CT study was negative or the initial baseline study did not lead to the diagnosis of a malignancy, repeat screening was performed after 12 months. A positive study prompted further workup according to an algorithm developed by I-ELCAP (16). A 3-month follow-up CT was done for nodules between 5 and $10 \mathrm{~mm}$, and, if growth was detected, appropriate diagnostic studies were done. If no growth was seen, an annual CT was scheduled. Growth was assessed visually by the radiologist comparing two CTs side by side on the workstation. FDG-PET was done for nodules $10 \mathrm{~mm}$ or larger or smaller nodules $(>7 \mathrm{~mm})$ showing growth, and, if positive, a percutaneous fine needle aspiration (FNA) or an intraoperative biopsy was performed. For nodules $10 \mathrm{~mm}$ or larger with benign characteristics, a 3-month follow-up CT was done instead of an FDG-PET.

\section{Spirometry}

FVC and $\mathrm{FEV}_{1}$ were measured with a computerized spirometer (Vmax 22; SensorMedics, Yorba Linda, CA). Results were expressed as a percentage of the predicted value according to the European Community Lung Health Survey (18). The presence and severity of airflow obstruction was determined following the criteria established by the Global Initiative for Chronic Obstructive Lung Disease (GOLD) (19).

\section{Data Analysis}

Normally distributed data were summarized as means (SD), and skewed data were summarized as medians (interquartile range). Data analysis was performed with SPSS for Windows, version 11 (SPSS, Inc., Chicago, IL).

\section{RESULTS}

\section{Study Population and Risk Factors}

A total of 911 (74\% men) subjects participated in the study. Their mean (SD) age was 54.7 (8.6) years. Their median (interquartile range) tobacco consumption was 30 (19-49.5) pack-years. Asbestos exposure was reported in only one subject. Other risk factors for lung cancer, such as radon, nickel, or chromium, were not reported.

\section{Low-Dose CT Findings}

In $440(48.3 \%)$ individuals, at least one pulmonary nodule was observed at baseline examination. Noncalcified nodules of any size were observed in 291 (31.9\%) subjects. At least one noncalcified nodule of $5 \mathrm{~mm}$ or more was found in $131(14.4 \%)$ subjects. Of the 291 individuals with noncalcified nodules, 164 $(56.4 \%)$ had one nodule, $70(24.1 \%)$ had two nodules, $31(10.7 \%)$ had three nodules, 11 (3.8\%) had four nodules, and $15(5.2 \%)$ had five or more nodules.

\section{Nodule Size and Attenuation}

A total of 973 nodules were detected on baseline low-dose CT studies (Table 1). Of these, 434 (44.6\%) were homogeneously calcified nodules and 539 (55.4\%) showed no calcification. Of the 539 noncalcified pulmonary nodules, 369 (68.5\%) had a maximum diameter less than $5 \mathrm{~mm}, 136(25.2 \%)$ were between 5 and $10 \mathrm{~mm}$, and $34(6.3 \%)$ were $10 \mathrm{~mm}$ or larger.

Of the 291 individuals with noncalcified pulmonary nodules, 160 (17.6\% of participants) only had nodules less than $5 \mathrm{~mm}$, 98 (10.7\% of participants) had at least one nodule between 5 and $10 \mathrm{~mm}$ (no nodules $>10 \mathrm{~mm}$ ), and 33 (3.6\% of participants) had at least one nodule of $10 \mathrm{~mm}$ or more (Table 2 ).

\section{FDG-PET for Nodules $10 \mathrm{~mm}$ or Larger or Smaller $(>7 \mathrm{~mm})$, Growing Nodules}

Twenty-four FDG-PET scans were done on 23 individuals (25 pulmonary nodules; Table 3 ). One individual had two FDG-PET scans (numbers 10 and 16, Table 3) for a nodule that grew from 10.5 to $14.5 \mathrm{~mm}$ in 7 months, and turned out to be a malignancy. The first FDG-PET was negative (false-negative), but the second showed an SUVmax of 1.6 (true-positive). Three of the nodules studied were slightly smaller than $10 \mathrm{~mm}$. Two were an $8-\mathrm{mm}$ nodule (number 25 , malignant) seen on the first annual repeat CT (new incidence nodule) and an 8.7-mm nodule (number 23, benign) seen on the baseline study in a patient in which the sputum cytology showed moderate to severe squamous metaplasia. FDG-PET scans for these two nodules were negative (one false-negative and one true-negative). A third 8-mm nodule (number 24, malignant) was seen in a patient with an additional 10-mm nodule (number 19, benign), which prompted the FDGPET study. Both nodules had negative FDG-PET scans (one false-negative, one true-negative), but the 8-mm nodule showed growth on follow-up CT (squamous cell carcinoma).

Altogether, 11 nodules were positive on FDG-PET, of which nine were malignant (median SUVmax, 1.8; interquartile range,

TABLE 1. DISTRIBUTION OF NODULES ACCORDING TO PRESENCE OF CALCIFICATION AND SIZE

\begin{tabular}{lcc}
\hline & NCNs (\% of all NCNs) & No. Calcified Nodules \\
\hline$<5 \mathrm{~mm}$ & $369(68.5)$ & \\
$5-10 \mathrm{~mm}$ & $136(25.2)$ & \\
$\geqslant 10 \mathrm{~mm}$ & $34(6.3)$ & \\
Total & $539(100 ; 55.4 \%$ of all nodules) & $434(44.6 \%$ of all nodules)
\end{tabular}

Definition of abbreviation: NCNs $=$ noncalcified nodules. 
TABLE 2. DISTRIBUTION OF INDIVIDUALS ACCORDING TO SIZE OF NONCALCIFIED NODULES

\begin{tabular}{lc}
\hline & $\begin{array}{c}\text { No. Individuals with NCNs } \\
\text { (\% of total cohort) }\end{array}$ \\
\hline$<5 \mathrm{~mm}^{*}$ & $160(17.6)$ \\
$5-10 \mathrm{~mm}^{\dagger}$ & $98(10.7)$ \\
$\geqslant 10 \mathrm{~mm}^{\ddagger}$ & $33(3.6)$ \\
Total & $291(31.9)$
\end{tabular}

For definition of abbreviation, see Table 1

* Individuals with only nodules $<5 \mathrm{~mm}$.

† With at least one nodule between 5-10 mm, no nodule $>10 \mathrm{~mm}$.

$\doteqdot$ With at least one nodule $\geqslant 10 \mathrm{~mm}$.

1.6-3.1), one was benign (SUVmax, 2.3, and necrosis on FNA with no growth on annual CT), and one was indeterminate (SUVmax, 1.2) because the patient refused further workup and was lost to follow-up. This indeterminate nodule was excluded from the analysis.

Fourteen nodules were negative on FDG-PET, of which four were malignant. These four malignancies were adenocarcinomas (two of them, numbers 15 and 25 on Table 3, had bronchoalveolar features in part of the tumor) and were all diagnosed in stage IA after growth was seen on short-term follow-up CTs. The sensitivity and specificity of FDG-PET to detect malignancy were 69 (95\% confidence interval, 41-89) and 91\% (95\% confidence interval, 63-99), respectively. Positive and negative predictive values were 90 (95\% confidence interval, 57-98) and 71\% (95\% confidence interval, 52-85), respectively.

\section{Lung Cancer Stages}

Lung cancer was detected in 14 (13 non-small cell lung cancers and 1 small cell lung cancer) of 911 individuals entered in the study. Eleven of the 13 non-small cell lung cancers and one small cell lung cancer were detected on the baseline study (prevalence, $1.32 \%)$. Of 424 individuals who have completed at least one annual repeat CT, two were diagnosed with non-small cell lung cancer (incidence, $0.47 \%)$. Of the prevalence cancers, $10(86 \%)$ were stage IA (T1N0M0) and one was stage IB (T2N0M0). The only small cell lung cancer was diagnosed in stage T3N3M0. The incidence cancers were both detected in stage IA (T1N0M0). Considering all cancers, 13 of $14(92.8 \%)$ were diagnosed in stage I.

\section{Invasive Procedures and Surgery}

There were no preoperative surgical biopsies performed. Six preoperative percutaneous FNAs were performed on four PETpositive nodules and two growing PET-negative nodules. Four of these were positive for malignancy and two were negative. One negative FNA (PET-positive) was followed up with a yearly CT showing no growth (false-positive PET). The possibility that this individual actually has a slow-growing cancer cannot be ruled out, although this is unlikely in view of the lack of growth. The other negative FNA (PET-positive) was inadequate because of a pneumothorax. This individual had an intraoperative biopsy showing a malignancy. The patient with small cell lung cancer was diagnosed with a bronchoscopy. The rest of the patients were diagnosed with intraoperative biopsies.

All patients with non-small cell lung cancer (prevalence

TABLE 3. NODULES STUDIED WITH F-18-FLUORODEOXYGLUCOSE POSITRON EMISSION TOMOGRAPHY

\begin{tabular}{|c|c|c|c|c|c|c|c|c|c|}
\hline No. & $\begin{array}{l}\text { Size on } \\
\text { CT }(m m)\end{array}$ & Location & CT Morphology & FDG Uptake & SUVmax & Follow-Up & Pathology & Stage & Result \\
\hline 1 & 20 & LLL & Solid & Positive & 2.3 & Lost & $?$ & & $?$ \\
\hline 2 & 19 & RUL & Solid & Positive & 1.2 & $1 \mathrm{yr}$ : no growth - lost & Necrosis & & $?$ \\
\hline 3 & 17.5 & RUL & Solid & Absent & & No growth & & & $\mathrm{TN}$ \\
\hline 4 & 17.2 & LUL & Solid & Absent & & No growth & & & $\mathrm{TN}$ \\
\hline 5 & 17 & RUL & Solid & Absent & & No growth & & & $\mathrm{TN}$ \\
\hline 6 & 16 & LUL & Solid & Positive & 1.6 & - & NSCLC (Squa) & T1N0M0 & TP \\
\hline 7 & 16 & LUL & Solid & Absent & & No growth & & & $\mathrm{TN}$ \\
\hline 8 & 15 & RUL* $^{*}$ & Solid & Positive & 5.7 & New nodule & NSCLC (Squa) & T1N0M0 & TP \\
\hline 9 & 15 & RLL & Solid & Positive & 1.5 & - & NSCLC (Aden) & T1N0M0 & TP \\
\hline 10 & 14.5 & $\mathrm{LUL}^{\dagger}$ & Nonsolid & Positive & 1.6 & 8 months: $4 \mathrm{~mm}$ growth & NSCLC (Aden) & T1N0M0 & TP \\
\hline 11 & 14 & RUL & Part-solid & Positive & 4 & - & NSCLC (Aden) & T1N0M0 & TP \\
\hline 12 & 13 & LLL & Solid & Absent & & No growth & & & $\mathrm{TN}$ \\
\hline 13 & 11.5 & LLL & Part-solid & Absent & & 5 months: $1.5 \mathrm{~mm}$ growth & NSCLC (Aden, 20\% BAC) & T1N0M0 & FN \\
\hline 14 & 11 & LUL & Solid & Positive & 2.2 & - & NSCLC (Undif) & T1N0M0 & TP \\
\hline 15 & 10.5 & LUL & Solid & Positive & 2 & - & NSCLC (Aden) & T2N0M0 & TP \\
\hline 16 & 10.5 & $\mathrm{LUL}^{\dagger}$ & Solid & Absent & & 12 months: $4 \mathrm{~mm}$ growth & NSCLC (Aden) & T1N0M0 & $\mathrm{FN}$ \\
\hline 17 & 10 & LUL & Solid & Positive & 1.8 & - & NSCLC (Squa) & T1N0M0 & TP \\
\hline 18 & 10 & RUL & Part-solid & Positive & 1.6 & - & Lymphoepitelioma & T1N0M0 & TP \\
\hline 19 & 10 & $\mathrm{RUL}^{\ddagger}$ & Solid & Absent & & No growth & & & $\mathrm{TN}$ \\
\hline 20 & 10 & RUL & Solid & Absent & & No growth & & & $\mathrm{TN}$ \\
\hline 21 & 10 & RUL & Solid & Absent & & No growth & & & $\mathrm{TN}$ \\
\hline 22 & 10 & LLL & Solid & Absent & & No growth & & & $\mathrm{TN}$ \\
\hline 23 & 8.7 & RLL & Solid & Absent & & No growth & & & $\mathrm{TN}$ \\
\hline 24 & 8 & $\mathrm{RUL}^{*}$ & Solid & Absent & & $\begin{array}{l}15 \text { months: } 3 \mathrm{~mm} \text { growth } \\
\text { and change in shape }\end{array}$ & NSCLC (Aden) & T1N0M0 & $\mathrm{FN}$ \\
\hline 25 & 8 & LUL* $^{*}$ & Part-solid & Absent & & New nodule & NSCLC (Aden, 80-90\% BAC) & T1N0M0 & $\mathrm{FN}$ \\
\hline
\end{tabular}

Definition of abbreviations: Aden = adenocarcinoma; BAC = bronchoalveolar carcinoma; CT = computed tomography; FDG = F-18-fluorodeoxyglucose; FN = false

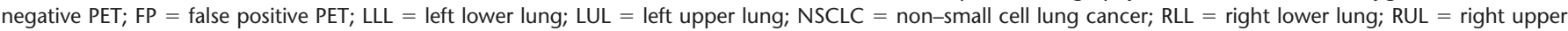

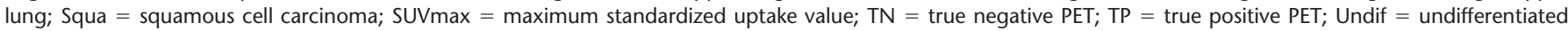
carcinoma.

* Incidence cancers.

† Same nodule on two different FDG-positron emission tomography (PET) scans (baseline and follow-up).

¥ Two different nodules on same FDG-PET scan. 
and incidence) underwent surgery. Eleven of $13(84.6 \%)$ had lobectomies and two $(15.4 \%)$ had wedge resections because of chronic obstructive pulmonary disease.

\section{Spirometry}

Of 834 subjects who had spirometry at baseline, $220(26.4 \%)$ had an $\mathrm{FEV}_{1} / \mathrm{FVC}$ of less than $70 \%$. Of these, $113(51.4 \%)$ had an $\mathrm{FEV}_{1}$ of less than $80 \%$ (mild airflow obstruction), 90 (40.9\%) had an $\mathrm{FEV}_{1}$ of less than 80 but at least $50 \%$ (moderate airflow obstruction), $14(6.4 \%)$ had an $\mathrm{FEV}_{1}$ of less than 50 but at least $30 \%$ (severe airflow obstruction), and three (1.4\%) had an $\mathrm{FEV}_{1}$ of less than $30 \%$ (very severe airflow obstruction). Of the 14 patients with non-small cell lung cancer, 11 (79\%) had airflow obstruction $\left(\mathrm{FEV}_{1} / \mathrm{FVC}<70 \%\right)$. Six had mild, four had moderate, and one had severe airflow obstruction.

\section{DISCUSSION}

Similar to recent reports $(9,20-22)$, this study confirms that a low-dose spiral CT-based protocol for early detection of lung cancer is useful in leading to early stage diagnosis in a high proportion of cases and may increase the chances for cure. Furthermore, this study shows that a carefully followed multidisciplinary diagnostic algorithm that includes FDG-PET minimizes unnecessary invasive procedures for benign lesions, without resulting in missed cancers caused by false-negative PET scans.

Lung cancer is one of the leading causes of death in the world. It is the most common cancer in many countries and, because most patients are diagnosed in late stages, it is also the most lethal (1-3). Less than $20 \%$ of individuals suffering this disease are diagnosed in stages in which curative surgery is an option (2). Survival rates for early-stage disease are quite high, approaching $80 \%$ in some series, but overall 5-year survival rates are approximately $15 \%$ (2). Five-year survival rates for patients who have already survived 2 years (conditional survival) remain significantly higher for those diagnosed in early stages, stressing the importance of early diagnosis (23).

Lung cancer screening with low-dose CT has shown to be effective in diagnosing lung cancer in the early stages. In ELCAP, $89 \%$ of lung cancers detected in the prevalence phase of the trial were diagnosed in stages I and II (9). In other screening trials, the proportion of cancers diagnosed in stages I and II varies between 75 and $90 \%(10-14)$. Whether this will result in a reduction in mortality from lung cancer has not been answered yet, and is being addressed in several trials underway. However, it is clear from a clinical point of view that lung cancer diagnosed and treated in early phases has a much better prognosis. Despite the fact that earlier screening trials with chest radiographs and sputum cytology failed to show a reduction in mortality, the results obtained from $\mathrm{CT}$ screening trials published thus far, and those presented herein, are strong enough to warrant more research in the field.

One of the major concerns regarding lung cancer screening with spiral CT is the rate of false-positive tests resulting in potentially harmful workups for benign diseases. The prevalence of noncalcified nodules on baseline low-dose spiral CT in different trials ranges between 19 and $69 \%(9,12,20-22)$. Limiting further workup to nodules of $5 \mathrm{~mm}$ or more has been shown to be appropriate by Henschke and coworkers (24) in a recent study reviewing diagnoses of lung cancer in a CT screening trial according to size. In a population of 2,897 participants, lung cancer was diagnosed only in individuals with nodules of at least $5 \mathrm{~mm}$ in diameter. Of 378 participants with the largest noncalcified nodule less than $5 \mathrm{~mm}$ in diameter, none was diagnosed with a malignancy. In our series, $32 \%$ of participants had noncalcified nodules of any size, but only $14 \%$ required further workup be- cause of the presence of at least one noncalcified nodule of $5 \mathrm{~mm}$ or more (positive CT scans). Among this group of individuals, the majority had small nodules between 5 and $10 \mathrm{~mm}$. Following these nodules with short-term follow-up CTs for detection of growth results in a low rate of invasive procedures for benign lesions. Larger nodules or nodules that show growth on followup CTs, all of which are more likely to be malignant, pose a more difficult problem and are more likely to result in unnecessary invasive procedures. Our findings suggest that the addition of FDG-PET to the protocol does not eliminate the risk of unnecessary invasive procedure completely, but may reduce it to a minimum. Excluding the patient with one nodule that was positive on FDG-PET but who was lost to follow-up, the specificity and positive predictive value of FDG-PET in our series was 91 and $90 \%$, respectively. What price, in terms of invasive procedures or even surgical interventions for benign lesions, is acceptable in screening protocols remains to be seen. In this series, no surgical intervention was done for a benign lesion, but one benign lesion did undergo an FNA. The risk for complications and the anxiety generated in the workup of benign lesions has to be considered as endpoints in future studies. On the other hand, using FDG-PET, the diagnosis of cancer in 9 of 13 patients was achieved 3 months earlier than if obtained by short-term follow-up CT. The implications this might have in terms of overall prognosis are unknown.

Although FDG-PET had a positive influence on the diagnostic management of 19 of 25 cases, the sensitivity and negative predictive value of FDG-PET for the diagnosis of malignant nodules in this population was 69 and $71 \%$. The overall sensitivity of FDG-PET for the diagnosis of pulmonary malignancies greater than $1 \mathrm{~cm}$ is over $95 \%$ (25). However, recent studies show that for pulmonary malignant nodules of less than $1 \mathrm{~cm}$ in size, the sensitivity of FDG-PET is very low (26). Four malignant nodules in our series did not show any FDG uptake. Two were smaller than $1 \mathrm{~cm}(8 \mathrm{~mm})$, and two only slightly larger (10.5 and $11.5 \mathrm{~mm}$ ). Furthermore, all four false-negative nodules were adenocarcinomas and two had a nonsolid component on the CT and bronchoalveolar features on the histologic analysis (Table 3 , nodule numbers 13 and 25). These characteristics (adenocarcinoma, part-solid component, and bronchoalveolar carcinoma) have all been reported by others as causes of false-negative nodules on FDG-PET scans (26). Therefore, it is the combination of FDG-PET with a short-term follow-up CT for negative studies that makes the screening algorithm useful. In addition, although the protocol calls for percutaneous FNAs of suspicious nodules (those $>10 \mathrm{~mm}$ or smaller nodules that grow), the results of this study support FDG-PET as a noninvasive substitute if caution in following up negative studies is taken. The optimal interval between a negative FDG-PET and a follow-up CT to detect growth remains to be determined. It may be longer that the 3month interval selected in this protocol, thus decreasing the potential hazards caused by radiation, as well as the costs of the algorithm.

SUVs may provide numeric thresholds to differentiate malignant and benign lesions, but in our series, visual assessment of FDG-PET scans classified the indeterminate noncalcified pulmonary nodules better. Visual analysis of FDG-PET scans was performed for diagnosis because it has been shown that SUV methodology is less straightforward than has often been assumed (27). Furthermore, the SUV of a lesion on FDG-PET decreases when the diameter is smaller than twice the spatial resolution of the system, which is 7 to $8 \mathrm{~mm}$ for our BGO scanner. In our study, if only lung nodules with an SUVmax greater than 2 would have been considered positive, FDG-PET would have contributed to establish the proper diagnosis in 14 cases instead of the 19 cases correctly diagnosed with visual analysis. The 
sensitivity and the negative predictive value of FDG-PET for the diagnosis of malignant nodules would have decreased to 23 and $52 \%$, respectively. The efficiency of a screening program will depend largely on the pretest probability (prevalence) of having the disease. The higher the prevalence, the lower the risk of false-positive results. The prevalence will be higher as the inclusion of individuals in the screening program becomes more selective, focusing on higher risk. One future possibility is the use of biomarkers, not only for early detection but for risk stratification as well (28). From a clinical point of view, the presence of airways disease may be important for risk stratification. In this study, $79 \%$ of the patients with lung cancer had airflow obstruction by GOLD criteria (19). Therefore, limiting enrollment to individuals with evidence of airflow obstruction might enhance efficiency. Related to this finding is the fact that $30 \%$ of the participants showed evidence of airflow obstruction. A recent preliminary randomized controlled lung cancer screening trial, in which patients were classified according to the presence of airways disease into high-risk (airflow obstruction) and moderate-risk (no airflow obstruction) individuals, showed that the probability of finding noncalcified nodules is significantly higher (40 vs. $22 \%$ ) in patients with airways obstruction (29). This also raises the issue of whether a screening program for lung cancer might be useful for screening other important diseases, such as chronic obstructive pulmonary disease, which is the fifth leading cause of death worldwide (30).

This report and others point out one very important aspect. Early lung cancer detection is complex and requires a multidisciplinary approach. With the data available to date, lung cancer screening, although promising, still requires intensive research before wide implementation. But it is quite clear that the diagnosis and management of lung cancer are due for a change. The need for a new staging system that addresses smaller lung cancers is emerging. Data from Europe and the United States strongly suggest that the current size threshold for staging lung cancers (i.e., $3 \mathrm{~cm}$ ) is not adequate and support the notion that detecting small-volume lung cancers provides a better chance for cure. The Spanish Bronchogenic Carcinoma Co-operative Group has found that classifying patients into four groups according to tumor size (0-2 cm, 2.1-4 cm, 4.1-7 cm, and $>7 \mathrm{~cm}$ ) better predicted prognosis (31). In the United States, using the 2003 Surveillance, Epidemiology, and End Results, or SEER, registry, Wisnivesky and coworkers (32) showed that 12-year survival among patients with stage I lung cancer who had undergone surgical resection was inversely proportional to the initial tumor size.

In conclusion, early detection of lung cancer after a carefully designed low-dose spiral CT-based protocol with the addition of FDG-PET for nodules of $10 \mathrm{~mm}$ or more, or for smaller $(>7 \mathrm{~mm}$ ) growing nodules, is possible. The addition of FDG-PET to the protocol may reduce unnecessary invasive procedures to a minimum without resulting in missed cancers. This strategy may improve chances for cure because epidemiologic data strongly suggest that the prognosis of lung cancer is significantly better when diagnosed in early stages.

Conflict of Interest Statement: G.B. does not have a financial relationship with a commercial entity that has an interest in the subject of this manuscript; M.J.G.-V. does not have a financial relationship with a commercial entity that has an interest in the subject of this manuscript; M.D.L. does not have a financial relationship with a commercial entity that has an interest in the subject of this manuscript U.M. does not have a financial relationship with a commercial entity that has an interest in the subject of this manuscript; W.T. does not have a financial relationship with a commercial entity that has an interest in the subject of this manuscript; N.S. does not have a financial relationship with a commercial entity that has an interest in the subject of this manuscript; A.C. does not have a financial relationship with a commercial entity that has an interest in the subject of this manuscript L.S. does not have a financial relationship with a commercial entity that has an interest in the subject of this manuscript; A.B.A. does not have a financial relation- ship with a commercial entity that has an interest in the subject of this manuscript; J.P. does not have a financial relationship with a commercial entity that has an interest in the subject of this manuscript; D.C. does not have a financial relationship with a commercial entity that has an interest in the subject of this manuscript; I.V. does not have a financial relationship with a commercial entity that has an interest in the subject of this manuscript; O.C. does not have a financial relationship with a commercial entity that has an interest in the subject of this manuscript P.D. does not have a financial relationship with a commercial entity that has an interest in the subject of this manuscript; P.S. does not have a financial relationship with a commercial entity that has an interest in the subject of this manuscript J.A.R. does not have a financial relationship with a commercial entity that has an interest in the subject of this manuscript; L.M. does not have a financial relationship with a commercial entity that has an interest in the subject of this manuscript; J.J.Z. does not have a financial relationship with a commercial entity that has an interest in the subject of this manuscript.

Acknowledgment: The authors thank Ms. Marta Moreno for her invaluable technical assistance.

\section{References}

1. Jemal A, Murray T, Samuels A, Ghafoor A, Ward E, Thun MJ. Cancer statistics, 2003. CA Cancer J Clin 2003;53:5-26.

2. Jemal A, Clegg LX, Ward E, Ries LA, Wu X, Jamison PM, Wingo PA, Howe HL, Anderson RN, Edwards BK. Annual report to the nation on the status of cancer, 1975-2001, with a special feature regarding survival. Cancer 2004;101:3-27.

3. Franco J, Perez-Hoyos S, Plaza P. Changes in lung-cancer mortality trends in Spain. Int J Cancer 2002;97:102-105.

4. Mulshine JL. Screening for lung cancer: in pursuit of pre-metastatic disease. Nat Rev Cancer 2003;3:65-73.

5. Melamed M, Flehinger B, Zaman M, Heelan R, Perchick W, Martini N. Screening for early lung cancer: results of the Memorial Sloan-Kettering study in New York. Chest 1984;86:44-53.

6. Fontana RS, Sanderson DR, Woolner LB, Taylor WF, Miller WE, Muhm JR. Lung cancer screening: the Mayo program. J Occup Med 1986;28: $746-750$.

7. Tockman M. Survival and mortality from lung cancer in a screened population: the John Hopkins study. Chest 1986;89:325S.

8. Kubik A, Polak J. Lung cancer detection: results of a randomized prospective study in Czechoslovakia. Cancer 1986;57:2428-2437.

9. Henshcke CI, McCauley DI, Yankelevitz DF, Naidich DP, McGuinness G, Miettinen OS, Libby DM, Pasmantier MW, Koizumi J, Altorki NK, et al. Early Lung Cancer Action Project: overall design and findings from baseline screening. Lancet 1999;354:99-105.

10. Swensen SJ, Jett JR, Sloan JA, Midthun DE, Hartman TE, Sykes AM, Aughenbaugh GL, Zink FE, Hillman SL, Noetzel GR, et al. Screening for lung cancer with low-dose spiral computed tomography. Am J Respir Crit Care Med 2002;165:508-513.

11. Diederich S, Wormanns D, Semik M, Thomas M, Lenzen H, Roos N, Heindel W. Screening for early lung cancer with low-dose spiral CT: prevalence in 817 asymptomatic smokers. Radiology 2002;222:773-781.

12. Pastorino U, Bellomi M, Landoni C, De Fiori E, Arnaldi P, Picchio M, Pelosi G, Boyle P, Fazio F. Early lung-cancer detection with spiral CT and positron emission tomography in heavy smokers: 2-year results. Lancet 2003;362:593-597.

13. Sone S, Li F, Yang ZG, Honda T, Maruyama Y, Takashima S, Hasegawa M, Kawakami S, Kubo K, Haniuda M, et al. Results of three-year mass screening programme for lung cancer using mobile low-dose spiral computed tomography scanner. Br J Cancer 2001;84:25-32.

14. Sobue T, Moriyama N, Kaneko M, Kusumoto M, Kobayashi T, Tsuchiya R, Kakinuma R, Ohmatsu H, Nagai K, Nishiyama H, et al. Screening for lung cancer with low-dose helical computed tomography: anti-lung cancer association project. J Clin Oncol 2002;20:911-920.

15. Gould MK, Maclean CC, Kuschner WG, Rydzak CE, Owens DK. Accuracy of positron emission tomography for diagnosis of pulmonary nodules and mass lesions: a meta-analysis. JAMA 2001;285:914-924.

16. Henschke CI, Yankelevitz DF, Smith JP, Miettinen OS. Screening for lung cancer: the early lung cancer action approach. Lung Cancer 2002; 35:143-148.

17. Zulueta JJ, Bastarrika G, García Velloso MJ, Lozano MD, Torre W, Pueyo J, Montes U, Gúrpide A, Campo A, García-López JJ, et al. Early lung cancer detection with low dose spiral computed tomography and positron emission tomography [abstract]. Am J Respir Crit Care Med 2004;169(Suppl):A752.

18. Roca J, Burgos F, Sunyer J, Saez M, Chinn S, Anto J, Rodriguez-Roisin R, Quanjer P, Nowak D, Burney P. References values for forced spirometry. Group of the European Community Respiratory Health Survey. Eur Respir J 1998;11:1354-1362. 
19. Pauwels RA, Buist AS, Calverley PMA, Jenkins CR, Hurd SS. Global strategy for the diagnosis, management, and prevention of chronic obstructive pulmonary disease: NHLBI/WHO Global Initiative for Chronic Obstructive Lung Disease (GOLD) workshop summary. Am J Respir Crit Care Med 2001;163:1256-1276.

20. Henschke C, Naidich D, Yankelevitz D, McGuinness G, McCauley D, Smith J, Libby D, Pasmantier M, Vazquez M, Koizumi J, et al. Early lung cancer action project: initial findings on repeat screenings. Cancer 2001;92:153-159.

21. Swensen SJ, Jett JR, Hartman TE, Midthun DE, Sloan JA, Sykes AM, Aughenbaugh GL, Clemens MA. Lung cancer screening with CT: Mayo Clinic experience. Radiology 2003;226:756-761.

22. Diederich S. Screening for early lung cancer with low-dose spiral computed tomography. Lancet 2003;362:588-589.

23. Merrill RM, Henson DE, Barnes M. Conditional survival among patients with carcinoma of the lung. Chest 1999;116:697-703.

24. Henschke CI, Yankelevitz DF, Naidich DP, McCauley DI, McGuinness G, Libby DM, Smith JP, Pasmantier MW, Miettinen OS. CT screening for lung cancer: suspiciousness of nodules according to size on baseline scans. Radiology 2004;231:164-168.

25. Schrevens L, Lorent N, Dooms C, Vansteenkiste J. The role of PET scan in diagnosis, staging, and management of non-small cell lung cancer. Oncologist 2004;9:633-643.
26. Nomori H, Watanabe K, Ohtsuka T, Naruke T, Suemasu K, Uno K. Evaluation of F-18 fluorodeoxyglucose (FDG) PET scanning for pulmonary nodules less than $3 \mathrm{~cm}$ in diameter, with special reference to the CT images. Lung Cancer 2004;45:19-27.

27. Herder GJ, Golding RP, Hoekstra OS, Comans EF, Teule GJ, Postmus PE, Smit EF. The performance of (18)F-fluorodeoxyglucose positron emission tomography in small solitary pulmonary nodules. Eur J Nucl Med Mol Imaging 2004;31:1231-1236.

28. Chanin TD, Merrick DT, Franklin WA, Hirsch FR. Recent developments in biomarkers for the early detection of lung cancer: perspectives based on publications 2003 to present. Curr Opin Pulm Med 2004;10:242-247.

29. Garg K, Keith RL, Byers T, Kelly K, Kerzner AL, Lynch DA, Miller YE. Randomized controlled trial with low-dose spiral CT for lung cancer screening: feasibility study and preliminary results. Radiology 2002;225:506-510.

30. Pauwels RA, Rabe KF. Burden and clinical features of chronic obstructive pulmonary disease (COPD). Lancet 2004;364:613-620.

31. Lopez-Encuentra A, Duque-Medina JL, Rami-Porta R, de la Camara AG, Ferrando P. Staging in lung cancer: is $3 \mathrm{~cm}$ a prognostic threshold in pathologic stage I non-small cell lung cancer? A multicenter study of 1,020 patients. Chest 2002;121:1515-1520.

32. Wisnivesky JP, Yankelevitz D, Henschke CI. The effect of tumor size on curability of stage I non-small cell lung cancers. Chest 2004;126: 761-765. 